\title{
Experimental Analysis of Receding Horizon Planning Algorithms for Marine Monitoring
}

\author{
Soo-Hyun Yoo, Andrew Stuntz, Yawei Zhang, Robert Rothschild, \\ Geoffrey A. Hollinger and Ryan N. Smith
}

\begin{abstract}
Autonomous surface vehicles (ASV) are becoming more widely used in environmental monitoring applications. Due to the limited duration of these vehicles, algorithms need to be developed to save energy and maximize monitoring efficiency. This paper compares receding horizon path planning models for their effectiveness at collecting usable data in an aquatic environment. An adaptive receding horizon approach is used to plan ASV paths to collect data. A problem that often troubles conventional receding horizon algorithms is the path planner becoming trapped at local optima. Our proposed Jumping Horizon (J-Horizon) algorithm planner improves on the conventional receding horizon algorithm by jumping out of local optima. We demonstrate that the J-Horizon algorithm collects usable data more efficiently than commonly used lawnmower patterns, and we provide a proofof-concept field implementation on an ASV with a temperature monitoring task in a lake.
\end{abstract}

\section{Introduction}

Autonomous surface vehicles (ASVs) are becoming more commonly used to collect data in oceans and inland waterways using instruments such as: acoustic doppler current profilers (ADCPs); conductivity, temperature, and depth sensors (CTDs); and sidescanning sonars. These autonomous vehicles allow data collection in tight places, such as in and around glaciers or ice, as well as in close proximity to land (e.g., around river deltas) (Curcio et al (2005), Grasmueck et al (2006)).

S. Yoo, Y. Zhang, G.A. Hollinger

Oregon State University, Corvallis, OR,

e-mail: $\quad$ yoos, zhanyawe $\quad$ @onid.oregonstate.edu, geoff.hollinger@

oregonstate. edu · A. Stuntz, R. Rothschild, R.N. Smith

Fort Lewis College, Durango, CO,

e-mail: \{abstuntz, rarothchild, rnsmith\}@fortlewis.edu 


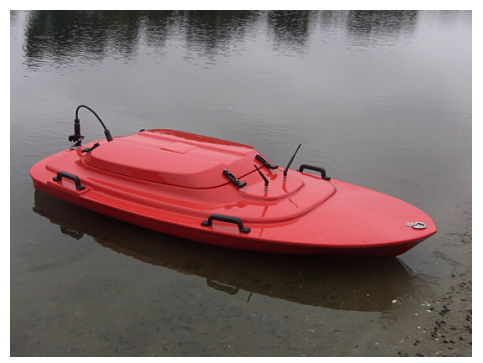

(a) Q-Boat $1800 \mathrm{P}$ with an integrated ADCP.

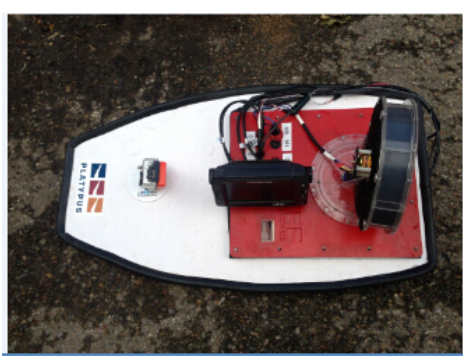

(b) Platypus Lutra with a dissolved oxygen and $\mathrm{pH}$ sensor.

Fig. 1 Two commercially-available autonomous surface vehicles for aquatic sampling.

However, commercially-available ASVs, such as the Platypus Lutra Figure 1(b), typically execute a simple lawnmower path to cover the area to be explored Figure 2. Such a path can provide high data yield, but at the expense of substantial fuel and time costs (Stoker et al (1996)).

We propose a receding horizon path-planning algorithm that, given an information or uncertainty map, generates a sampling path to maximize the information gathered or reduce the uncertainty. We compare this algorithm against a simple lawnmower path planner for a given transport budget and examine the effects of various algorithm parameters on the quality of the generated path. Furthermore, we propose a Jumping Horizon (J-Horizon) algorithm that improves on the conventional receding horizon algorithm by varying the look ahead step size if desired threshold values cannot be found within the current horizon. This allows the planner to "jump" out of local optima if higher peaks can be found elsewhere on the map. Finally, we validate our simulated results using an ASV to collect an initial data set. The J-Horizon algorithm is then run over the scalar field produced from the initial data and a qualitative analysis is given. The J-Horizon planner is able to produce paths superior to a simple lawnmower pattern in simulation and experimentally it is shown that the path is able to cover more area and generate a better quality scalar field.

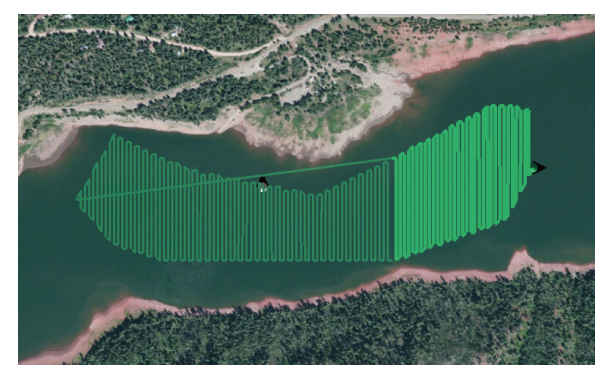

Fig. 2 The proprietary area search algorithm from Platypus generates a dense lawnmower pattern that is highly energy-inefficient. 


\section{Related Work}

Past work has shown that a receding horizon path planner is effective at optimizing paths in "no-fly" zone environments with hard constraints, as shown by Schouwenaars et al (2004), where the agent is prohibited from entering certain areas bounded by walls. This is a useful constraint for aerial and land vehicles that must navigate cluttered environments. However, these constraints do not apply to an ASV that must cover a large body of water such as a lake or the open ocean.

In previous work, AUVs have played a similar role as the ASV in our project. Binney et al (2010) describe an offline path planner for an uncertainty area. Hollinger and Singh (2008) describe an approach for multiple agents searching for a target in a known environment. Hitz et al (2014) discuss a path planner that can choose an efficient path for measurement of fluorescent bacteria in the ocean using an ASV. To reduce computational complexity, all of these authors employ a receding horizon path planner. Besides on ASVs, receding horizon algorithms are widely used in other robotics scenarios. Tisdale et al (2009) describe a receding horizon path planner for multiple unmanned aerial vehicles to search for a stationary object. However, none of them examine the effect of the horizon length or the possibility of modifying this horizon based on the remaining information.

Frolov et al (2014) compare lawnmower paths to other planning algorithms using fleets of research vehicles. They come to the conclusion that lawnmower paths are only marginally worse than adaptive algorithms. They also conclude that graphbased search algorithms are actually worse than lawnmower patterns since they are unable to adapt to prior uncertainty they cannot maximize their performance. Our J-Horizon algorithm adapts to the environment and removes these limitations to provide improved performance.

Gotovos et al (2013) propose a Level Set Estimation (LSE) algorithm that uses Gaussian Processes to estimate level sets of measured quantities and generate sampling points that reduce uncertainty around a certain threshold level. In a different context, Hollinger and Sukhatme (2013) describe an incremental sampling-based motion planning algorithm. Instead of reducing the uncertainty, they try to optimize the information gathering, depreciating the information value of sampled points.

A key limitation of existing research in receding horizon planning is that none of the aforementioned works discusses the role of parameters in the receding horizon algorithm. In addition, prior research has not focused on a single ASV performing data collection over large areas. In this paper, we address this gap in research in the aforementioned papers through the presentation of the J-Horizon algorithm. We present the application of our proposed method over different scalar fields both in simulation and in field experiments. The algorithm's performance is experimentally demonstrated to outperform existing lawnmower and receding horizon methods. 


\section{Problem Setup}

\subsection{Objective Function}

Often when sampling in an aquatic environment, scientists plan paths that follow a basic lawnmower pattern or spiral pattern. Previous work has looked at the difference between sampling in a lawnmower and a spiral pattern (Mora et al (2013)). This prior study takes into account the energy consumed by the autonomous vehicle and shows that a systematic spiral pattern collects more informative data and uses less energy than a lawnmower pattern.

In this paper, the J-Horizon planner addresses the following maximization problem:

$$
p^{*}=\underset{p \in \psi}{\arg \max } R(p) \quad \text { s.t. } \quad c(p) \leq B
$$

where $\psi$ is the space of possible trajectories for the ASV, $B$ is the initial budget (e.g., time, fuel), and $I$ is a function that represents the reward of information gathered or uncertainty reduced along the path $p$. In order for the algorithm to plan over a scalar field, a small amount of pilot data is required. This data can than be modeled or expanded, and then the algorithm can be run over an estimated scalar filed to produce paths.

\subsection{Experiment Setup}

We first present a simulation setup that uses generated scalar fields to compare performance between J-Horizon, conventional receding horizon, and lawnmower planning algorithms. We also use a real-world dataset acquired from Lake Haviland outside of Durango, $\mathrm{CO}$ to generate a path maximizing gathered information for a given transport budget.

\subsubsection{Simulation}

The J-Horizon algorithm is most effective when there is a prior dataset that can be used to generate an information map. The reward function is then specified by the maximum amount of new information that could be gathered at a map location. Furthermore, the algorithm improves upon the conventional receding horizon algorithm by seeking out areas of high reward when the local map area has been exhausted of new information, resulting in its "jumping" behavior.

A MATLAB script was used to generate 2960 different scalar fields with varying numbers and distributions of reward peaks. Between 5 and 50 such peaks were 
randomly generated on each map with a reward value that decays as a function of distance from the peak center. One such field is visualized in Figures 4 and 5 as contour maps.

The total reward accumulated along a path generated by J-Horizon for a given fuel budget was averaged for these scalar fields. This performance was compared with that of a lawnmower exploration pattern on the same datasets and fuel budget.

\subsubsection{Hardware}

The Platypus Lutra was used to take physical samples from Lake Haviland. It is a fan-powered ASV, maneuverable and capable of sampling data in lakes or other small bodies of water. The ASV is small and is an ideal choice on which to test our algorithm with limited sensing capabilities in a relatively large body of water. The ASV samples temperature, conductivity, $\mathrm{pH}$, and $\mathrm{DO}$, and includes a side scanning sonar. This allows it to map the bottom of the lake and measure depth.

The Platypus Lutra ASV has non-holonomic constraints that limit its ability to execute some of the sharper turns produced by the J-Horizon algorithm. Thus, due to hardware limitations, it is necessary to modify the path produced by J-Horizon. These modifications allow the ASV to follow the planned path. Due to the limited locomotion of the Platypus Lutra as well as a need to simplify data collection, some assumptions have to be made:

1. The ASV is limited in its motion and has non-holonomic turning constraints.

2. That sampled scalar fields were not dramatically changing over time.

3. Distance traveled equates to using a linear and constant amount of energy.

4. Additional data sampling points at a given location correlates to better quality data.

\section{Algorithm Design}

We seek to maximize the reward function for a given transport budget. In reality, this budget is a combination of fuel expenditure, time, and distance, each of which are specific to the vehicle and data collection scheme in use. For simplicity, we assume these factors are linearly related and that acceleration (e.g., due to turning, data collection) has zero cost.

In addition, we enhance the conventional receding horizon algorithm by increasing the lookahead step size if none of the predicted future states satisfy a reward threshold, allowing the planner to "jump" out of low-information areas. This makes J-Horizon especially effective when the input scalar field has high local variability.

The sequence of potential future steps, as well as the final generated path, are stored in a tree wherein each node stores the state of the ASV, which consists of the cumulative reward value of the path, remaining budget, and the location of the 
ASV. Each lookahead step recursively generates a number of possible future states. Of these, the best branch is chosen, and the rest are pruned. The sequence of nodes remaining after the remaining budget reaches zero is considered the optimal path.

The lawnmower and J-Horizon algorithms share the same functions to calculate the information available at a map location and to depreciate the available information after sampling that location.

The following sections describe the J-Horizon implementation shown in Algorithms 1,2 , and 3 by line number.

\subsection{Algorithm 1 - Main}

Path planning begins with the specified transport budget $B$ and loops over the following four steps until either the budget is expended or the planner becomes stuck:

6: From the current state $\sigma$, take $L$ lookahead steps with LOOKAHEAD. This updates the path tree with possible future states $L$ levels below the current node.

7: Find the location of the "best" adjacent node that will achieve the highest reward at the end of $L$ steps through that node.

8: Prune the path tree of all descendants under the current node.

9: Add sample point nodes between current and best locations and update the current node to the latest node.

\subsection{Algorithm 2 - Lookahead}

Given an initial state $\sigma$ and maximum recursion depth $d$, we recursively generate and add possible future states to the path tree. Each step is taken with a new, temporary copy of all data. During each call, it performs the following:

1: Generate set of future states $S_{f}$ from $\sigma$.

3: Remove a fraction $R \sim U([0,1))$ of the states (but not all) in $S_{f}$.

7: Recurse on each descendant node.

\subsection{Algorithm 3 - J-Horizon}

Given a state $\sigma$ and an information threshold $t$, probe outwards from the given location and update the map:

2: Start with a sample interval of $D$.

3: Calculate number of future states to generate $b$ per some factor $F$.

4: While $S_{f}$ is empty, perform the following: 
5: Generate $b$ equally spaced points around a circle of radius $D$ around $\sigma$.

6: For each such point, if the quality of the map at that point exceeds $t$, then add the point to $S_{f}$.

8-9: Increment $\delta$ by $D$ and update $b$.

10: Update the information map.
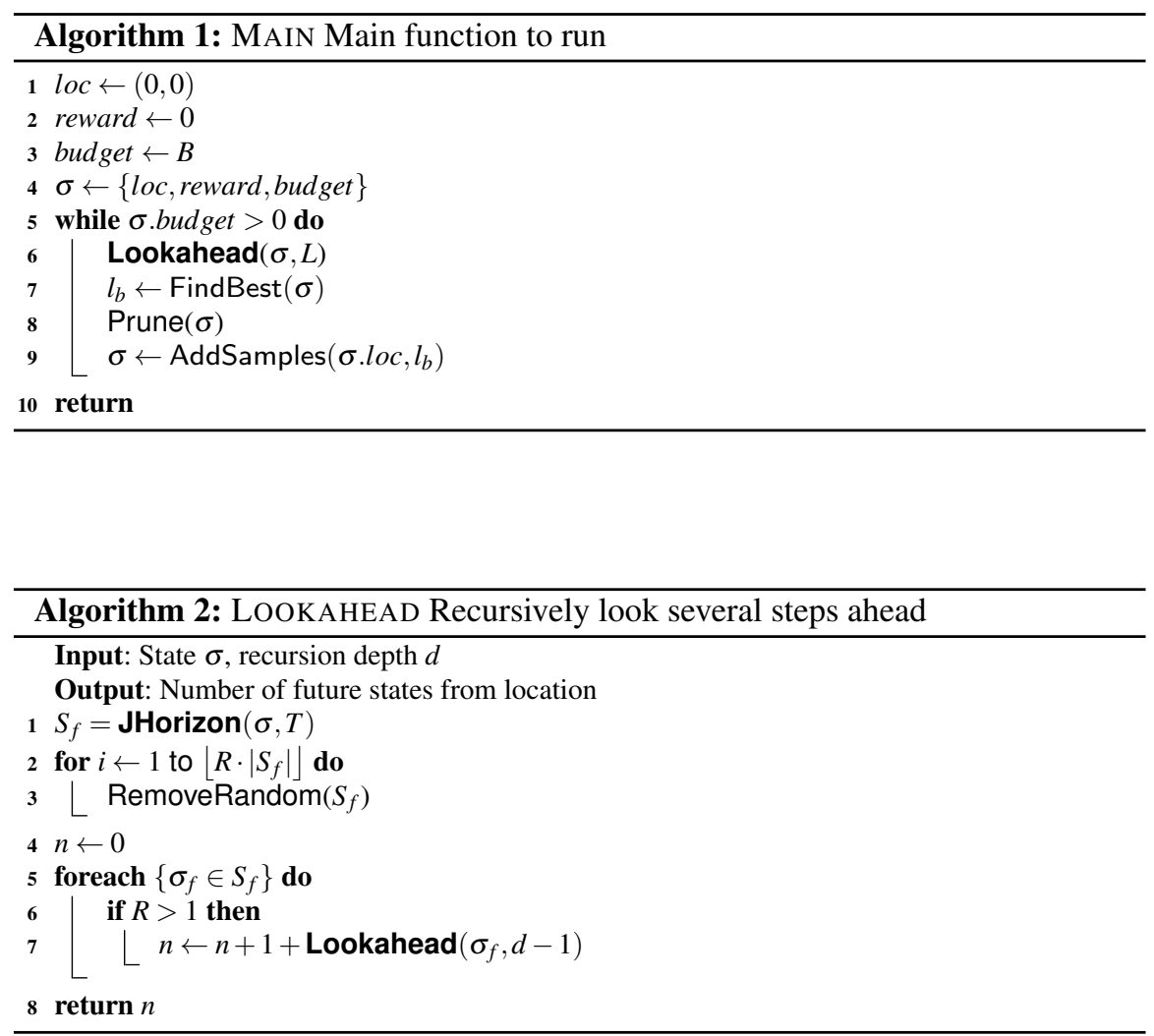

\section{Results}

In this section, we present the results of application of the J-Horizon algorithm on simulated data, as well as data collected during a field trial. 


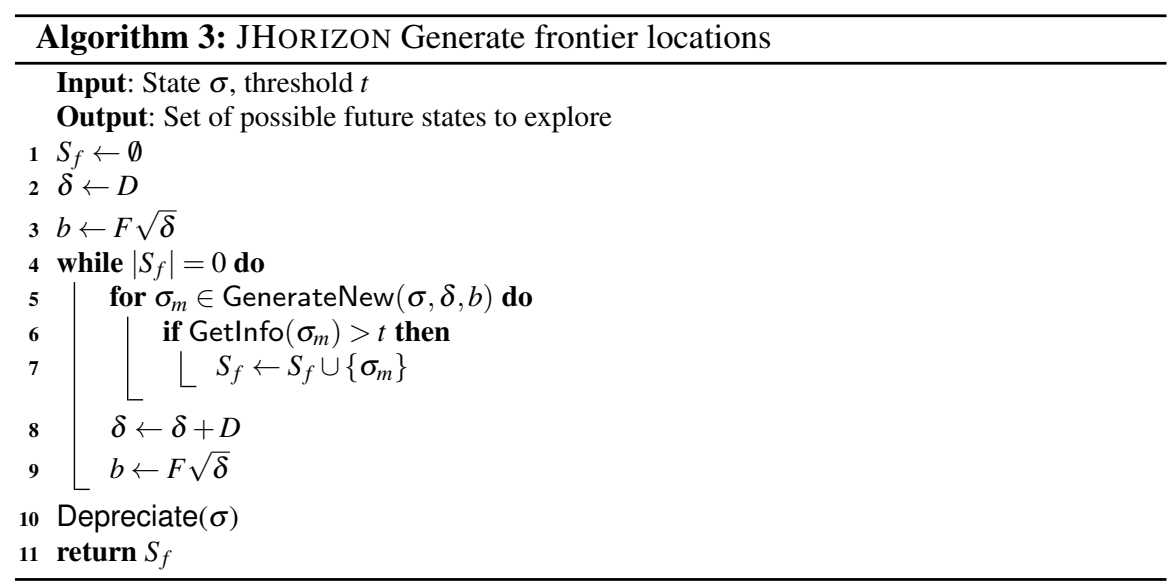

\subsection{Simulation Results}

2960 simulated scalar fields were generated for testing and validation of the JHorizon path planner. We compare the quality of the paths generated by the JHorizon planner to that of a simple lawnmower pattern with the same transport budget. The quality metric is the sum of the data collected over the course of the path. For the purpose of comparison, the information gathered was arbitrarily selected to correspond to the deviation from a particular target value of the quantity of interest (as seen in Figure 3). By identifying pertinent or interesting data, the algorithm is able to successfully maximize data collection for a given deployment region. Such quantities could represent the uncertainty in temperature or any other data set that can be approximated by a scalar field.

The simulated vector field seen in Figure- 3 is representative of many types of scalar data. One of the benefits of the J-Horizon algorithm is that it can plan across any type of scalar field: temperature, humidity, pressure, any kind of data that can be represented as a scalar field. The point being that the user may specify the data being looked for and J-Horizon will attempt to maximize the data collection. Figure 3 is an example of J-Horizon planning in high areas, whether those areas are high temperature, pressure, etc.

Figures 4, 5(a), and 5(b) show a typical lawnmower, receding horizon, and JHorizon path, respectively, planned over a simulated scalar field. The same transport budget was used for all three paths, yet the quality of the paths were 132, 189, and 420, respectively. The J-Horizon planner outperforms lawnmower by a factor of 3.18. Lawnmower required 3.51 times the transport budget to achieve the same reward on the same map and still fails to bring the maximum uncertainty below the threshold of 0.1 .

Figure 6(a) compares the information gathering ability of the three algorithms with increasing budget. J-Horizon gathers information most rapidly. When the bud- 


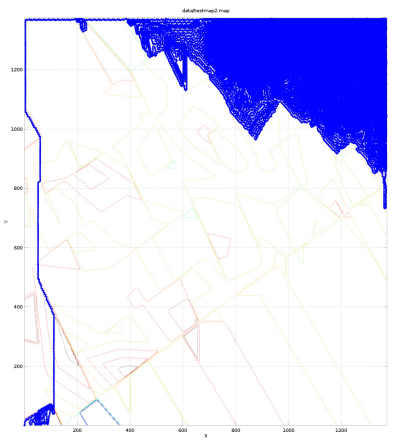

(a) Dense path planned over reward field.

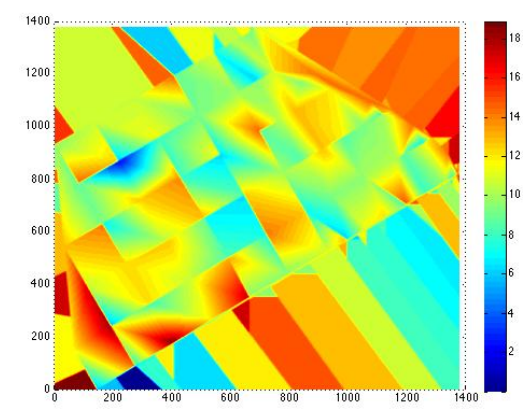

(b) Field reward level map. Red indicates high reward.

Fig. 3 J-Horizon path planned over simulated scalar field

get is large enough, the information gathered by the receding horizon planner is nearly equivalent to that of the J-Horizon planner.

Figure 6(b) shows the information gathering ability of the J-Horizon planner against a lawnmower pattern as we decrease the fraction of generated future states. For example, JH80\% indicates the algorithm generates $80 \%$ of the usual number of future states. Even at JH20\%, J-Horizon significantly outperforms a lawnmower path. This suggests it is possible to drastically reduce computational complexity with only a minor performance penalty.

One of the most advantageous qualities of this planner is that it is not limited to any particular search space. It is capable of planning paths over anything that can be estimated by a scalar field.

\subsection{Experimental Results}

Here, we present results from field trials for the implementation of the J-Horizon planner over a scalar field of surface temperature in a small lake in Colorado ${ }^{1}$. Specifically, the goal presented to the ASV is to sample at low-temperature regions, which corresponds to information reward in this case. We use the Platypus Lutra ASV, as shown in Figure 1(b), to conduct an initial survey and then use the J-Horizon planner to compute a new path with the objective to minimize the uncertainty and maximize information gain on the underlying scalar field. The initial path for representative data collection is presented in Figure 7(a), with the scalar field generated from these data and the path planned by the J-Horizon planner shown in

\footnotetext{
${ }^{1}$ The specific location of the field trials is Lake Haviland, outside of Durango, CO, located at $37^{\circ}$ $31^{\prime} 55^{\prime \prime} \mathrm{N} 107^{\circ} 48^{\prime} 27^{\prime \prime} \mathrm{W}$.
} 


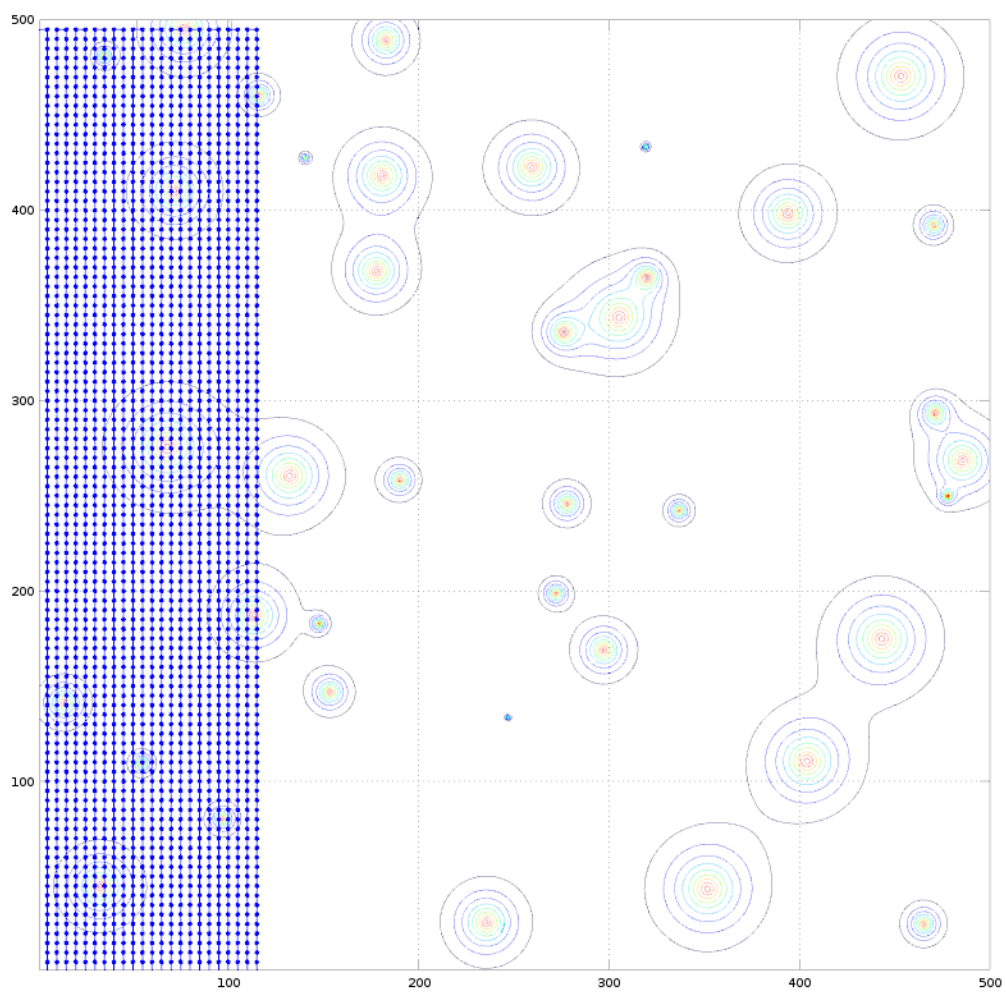

Fig. 4 Lawnmower path on generated scalar field. Reward of 132.

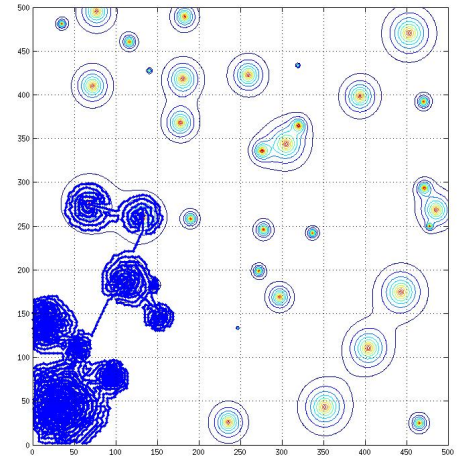

(a) Path generated by receding horizon planner. Note the path lingers in the high-reward area at the lower left for a long time before moving on to more worthwhile areas. Reward of 189 .

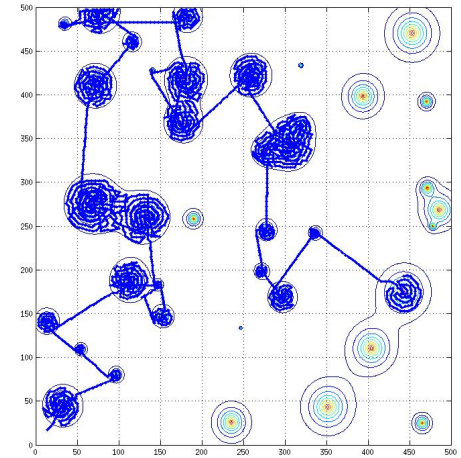

(b) Path generated by J-Horizon planner with threshold of 0.1 . Once the peak at the lowerleft has been exhausted of potential reward, the planner quickly moves on to other points of interest. Reward of 420.

Fig. 5 Effect of reward threshold on J-Horizon jumping behavior 


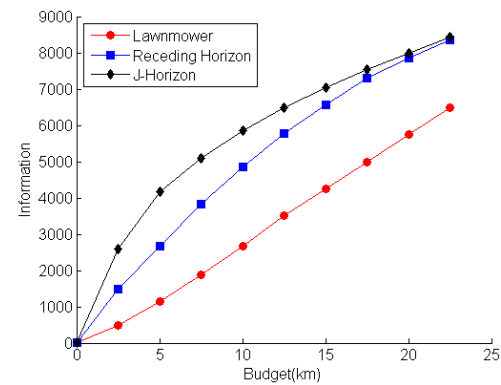

(a) Information gathered with increasing budget.

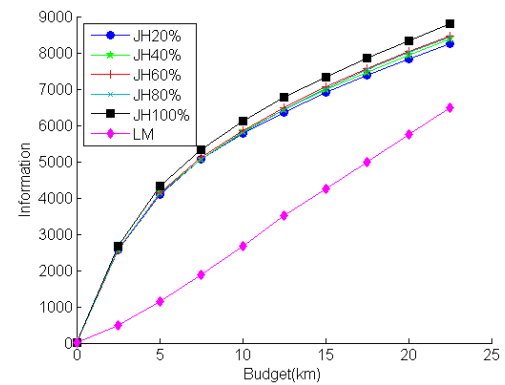

(b) The information gathering ability of the $\mathrm{J}$ Horizon planner against a lawnmower pattern as we decrease the fraction of generated future states.

Fig. 6 Performance comparison between lawnmower, receding horizon, and J-Horizon algorithms

Figure 7(b). The ASV executed the path prescribed by the J-Horizon planner, and results are shown in Figure 8.

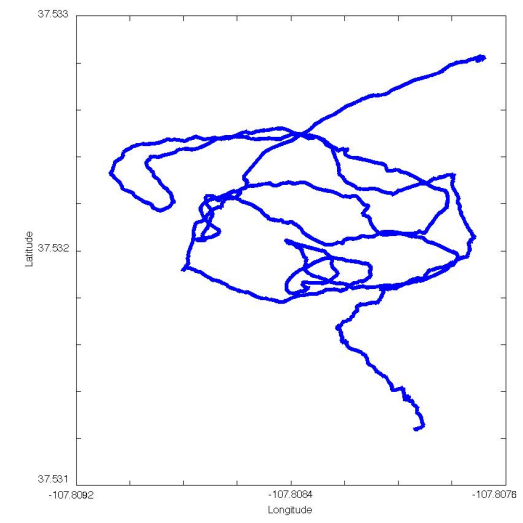

(a) The ASV's initial path on Lake Haviland outside Durango, CO.

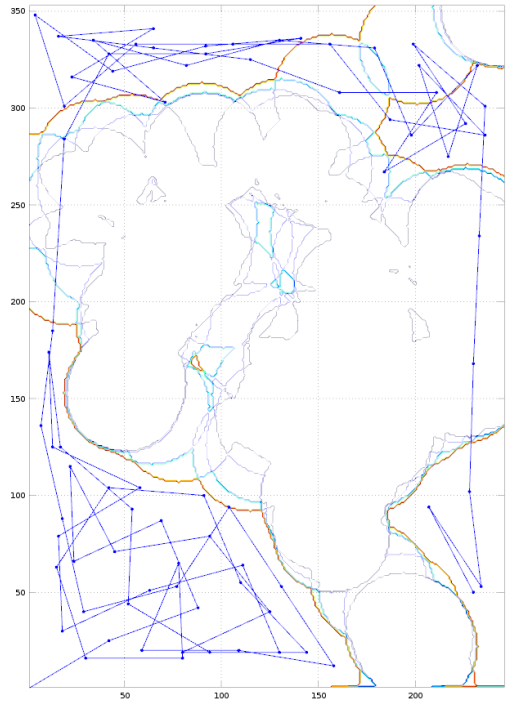

(b) J-Horizon path generated on ASV path shown in Fig. 7(a).

Fig. 7 Paths executed by the ASV to test and demonstrate the J-Horizon planner.

As seen in Figure 7(b), the J-Horizon gathers data in areas of low data yield from the initial data collection. For instance, the lower left hand corner of Figure 7(b) is 


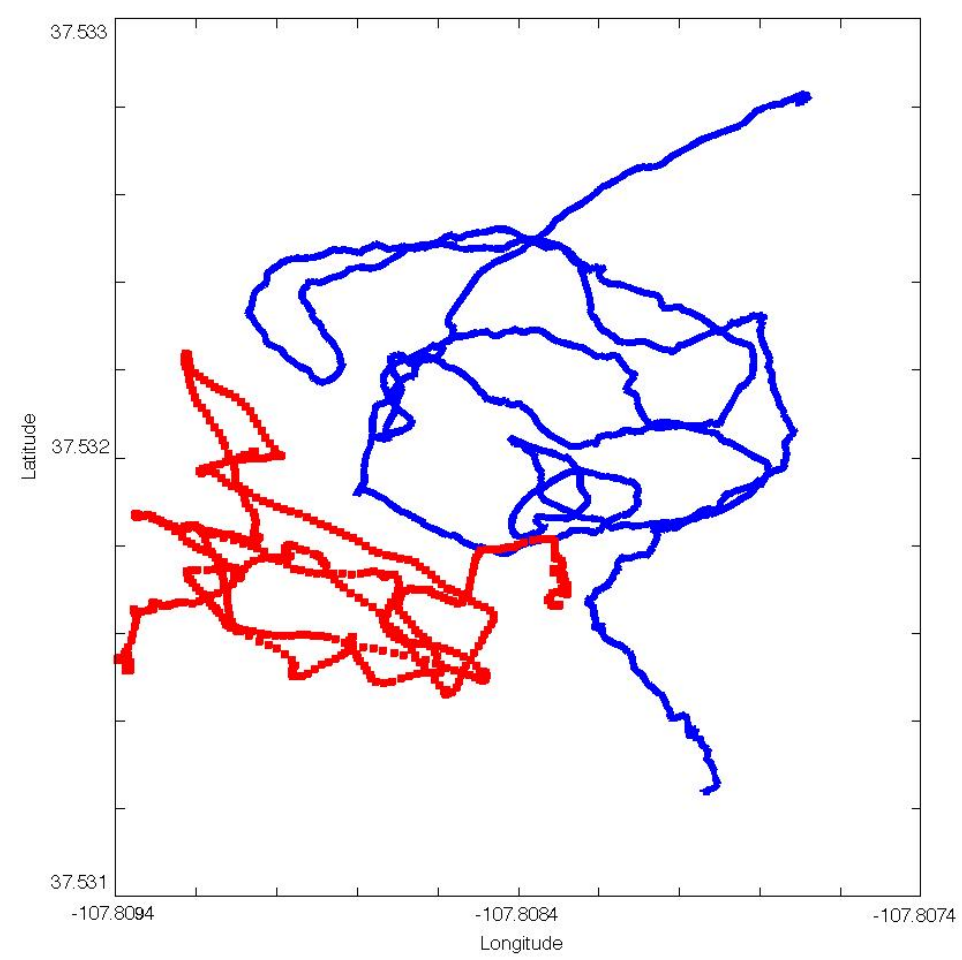

Fig. 8 The initial, data collection path (upper, blue) and the J-Horizon path (lower left, red) executed by the ASV on Lake Haviland.

an area that was not covered in the initial survey and requires more data collection to accurately represent the underlying field. This is the area of focus for our execution of the J-Horizon planner path, as more than half of entire length of the planned path lies within this region. The portion of the planned path that was executed is shown in Figure 8 by the red path.

\section{Conclusion}

Improved algorithm design for autonomous vehicles operating on water has a promising future in robotics. Collecting higher quality data that can be better utilized by scientists, as well as reducing costs of the data collection, is a key goal in making autonomous monitoring a reality. In this paper, we presented a receding horizon algorithm that attempts to find an optimized path to perform costly, and 
sometimes difficult or dangerous, data collection in oceans or other large bodies of water. In conclusion, we have presented a novel approach to better collect data over scalar fields. Simulation results show a $14.53 \%$ gain in reward of information collection compared to a lawnmower pattern while in simulation. The J-Horizon planner shows a $23.85 \%$ increase in information gathered in a simple experimental trial compared to a lawnmower pattern. Both of these results show quality gains compared to a lawnmower path of equivalent length. These optimized sampling paths allow scientists to more easily collect pertinent data in the field.

\section{Future Work}

Extended hardware trials would verify that the simplifying assumptions made in the algorithm design are realistic. Additional performance improvements could be made by running the planning algorithm on the ASV to update the error of the scalar field and re-plan in realtime. This would serve to allow the ASV to run autonomously in highly dynamic environments for longer periods of time without having to transmit data to the shore for processing.

The obvious extension of this work is the application to Autonomous Underwater Vehicles, and sampling in three dimensions. After further testing and validation on 2-D scalar fields, we are planning to investigate problems that exist for both underwater and aerial applications.

Finally, we are investigating an extension to the J-Horizon planner that includes applications for frontier searching, enabling a robotic platform to explore areas with unknown data quality. Such an algorithm will aim to balance explore vs. exploit in missions, searching new areas while also collecting data in areas that are deemed interesting or have low data density.

\section{Acknowledgment}

The authors would like to thank Jonathan Nash at Oregon State University for his insightful comments and insight into the algorithmic development. A. Stuntz was supported in part by ONR grant N00014-14-1-0490. R.N. Smith, A. Stuntz and R. Rothschild were supported by NSF Grant -DUE- 1068341, a gift from the Fort Lewis Foundation, and a Fort Lewis College Deans award for innovative research. S. Yoo, Y. Zhang, and G. Hollinger were supported in part by ONR grant N0001414-1-0509. 


\section{References}

Binney J, Krause A, Sukhatme G (2010) Informative path planning for an autonomous underwater vehicle. In: IEEE International Conference on Robotics and Automation(ICRA)), Anchorage, Alaska, pp 4791-4796

Curcio J, Leonard J, Patrikalakis A (2005) Scout - a low cost autonomous surface platform for research in cooperative autonomy. Oceans pp 725-729

Frolov S, Garau B, Bellingham J (2014) Can we do better than the grid survey: Optimal synoptic surveys in presence of variable uncertainty and decorrelation scales. Journal of Geophysical Research: Oceans 119(8):5071-5090, DOI 10.1002/ 2013JC009521, URL http: / / dx . doi . org/10 . 1002 / 2013 JC0 09521

Gotovos A, Casati N, Hitz G, Krause A (2013) Active learning for level set estimation. In: Interational Joint Conference on Artificial Intelligence, Beijing, China

Grasmueck M, Eberli GP, Viggiano DA, Correa T, Rathwell G, Luo J (2006) Autonomous underwater vehicle (auv) mapping reveals coral mound distribution, morphology, and oceanography in deep water of the straits of florida. Geophysical Research Letters 33(23)

Hitz G, Gotovos A, Pomerleau F, Garneau ME, Pradalier C, Krause A, Siegwart R (2014) Fully autonomous focused exploration for robotic environmental monitoring. In: Robotics and Automation (ICRA), 2014 IEEE International Conference on, pp 2658-2664, DOI 10.1109/ICRA.2014.6907240

Hollinger G, Singh S (2008) Proofs and experiments in scalable, near-optimal search by multiple robots. In: Robotics: Science and Systems, June, 2008

Hollinger GA, Sukhatme G (2013) Sampling-based motion planning for robotic information gathering. Robotics: Science and Systems

Mora A, Ho C, Saripalli S (2013) Analysis of adaptive sampling techniques for underwater vehicles. Autonomous Robots 35(2-3):111-122

Schouwenaars T, How J, Feron E (2004) Receding horizon path planning with implicit safety guarantees. In: American Control Conference, 2004. Proceedings of the 2004, IEEE, vol 6, pp 5576-5581

Stoker C, Barch D, Farmer J, Flagg M, Healy T, Tengdin T, Thomas H, Schwer K, Stakes D (1996) Exploration of mono lake with an rov: a prototype experiment for the maps auv program. Autonomous Underwater Vehicle Technology AUV'96:33-40

Tisdale J, Kim Z, Hedrick JK (2009) Autonomous path planning and estimation using uavs. IEEE Robotics and Automation Magazine 16(2):35-42 